\title{
A Very Accurate Measurement System for Multielectrode Capacitive Sensors
}

\author{
Ferry N. Toth, Gerard C. M. Meijer, and Harry M. M. Kerkvliet
}

\begin{abstract}
A very accurate capacitance-measurement system consisting of a discrete capacitance-dependent oscillator and a microcontroller has been developed. It can measure multielectrode capacitors with capacitances up to $2 \mathrm{pF}$, with an accuracy of $100 \mathrm{ppm}$ with respect to a reference capacitor. The resolution amounts to $50 \mathrm{aF}$ with a total measurement time of $300 \mathrm{~ms}$.
\end{abstract}

\section{INTRODUCTION}

$\mathbf{T}$ HE capacitance-measurement system presented here is based on earlier work [1]. The previous design involved a linear capacitance-controlled oscillator (the so-called modified Martin oscillator) that allowed several capacitors and an offset capacitance to be measured in exactly the same way. By applying continuous auto-calibration, gain and offset errors were reduced to an insignificant level. However, the previous system had two major disadvantages when applied in capacitive sensor systems.

First, it only measured capacitances with a common electrode. More advanced multi-electrode structures, which are often required in capacitive position sensors [2]-[4], call for multiplexing on both sides of the capacitances. Unfortunately, adding an additional multiplexer at the input of the system can disturb the continuous auto-calibration scheme, since the parasitic capacitances must not change during the measurement cycle.

Second, it could only measure accurately over a $50 \mathrm{fF}$ range. With a $2 \mathrm{pF}$ range the accuracy degraded to $0.4 \%$. Applications in accurate weighing equipment, angular encoders [5] and position sensors require a much higher accuracy over a $1 \mathrm{pF}$ range.

The new circuit design implements double-sided multiplexing and also improves the linearity by more than one order of magnitude. The concepts presented in [1] and [6] are used to reduce the offset, the gain and the effect of the capacitance of the connecting cables to an insignificant level. The circuit can be integrated on a chip or realized using readily available components, which allows an economical production of both small and large series.

Manuscript received April 24, 1995; revised December 26, 1995. This work was sponsored by Enraf BV and STW, the Duth Technology Foundation.

The authors are with the Faculty of Electrical Engineering-DIMES, Delft University of Technology, Mekelweg 4, 2628 AG Delft, The Netherlands.

Publisher Item Identifier S 0018-9456(96)03514-0.

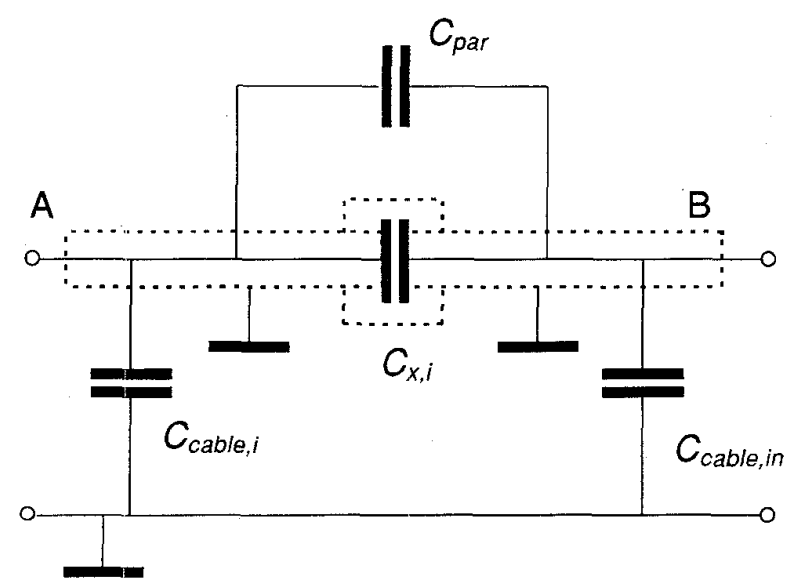

Fig. 1. Elimination of parasitic capacitances.

\section{BASIC PRINCIPLES}

The system is based on a capacitance-controlled oscillator. Because a microcontroller is used to measure the period and to control the multiplexer, several capacitances can be measured sequentially. A number of measures have been taken to minimize the effect of the main nonidealities. Each of these will be discussed in turn below.

\section{A. Shielding}

Several parasitic capacitances can be seen in Fig. 1. As will be shown below, the effects of cable capacitances $C_{c a b l e, i}$ and $C_{c a b l e, \text { in }}$ will be reduced to an insignificant level. However, since the parasitic capacitor $C_{p a r}$ is parallel to the measurand $C_{x, i}$ it cannot be eliminated electronically. Still it can be sufficiently reduced by shielding the terminals $\mathrm{A}$ and $\mathrm{B}$.

\section{B. Two-Port Measurement}

Although shielding reduces $C_{p a r}$, it greatly increases cable capacitances $C_{c a b l e, i}$ and $C_{c a b l e, i n}$. It is useful to look at the capacitance as a two-port (Fig. 1). In that case, the effect of $C_{\text {cable, } i}$ can be eliminated by connecting an ideal voltage source to terminal $\mathrm{A}$, and $C_{c a b l e, \text { in }}$ can be eliminated by connecting an ideal current meter to terminal B. In both cases, the cable capacitances are effectively shorted.

A practical circuit is shown in Fig. 2. Here the NANDgates operate as low-impedance voltage sources with on/off switches. The op amp operates together with $C_{f}$ as a lowimpedance charge amplifier. The capacitance $C_{o s}$ results from 


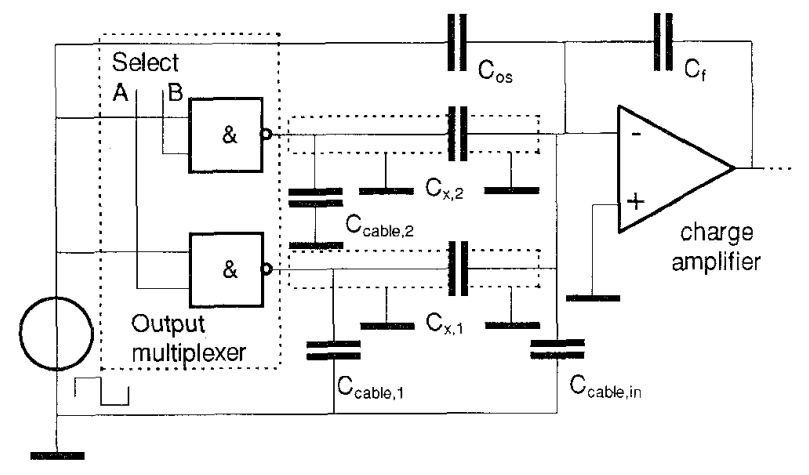

Fig. 2. The input stage of the capacitance-controlled oscillator:

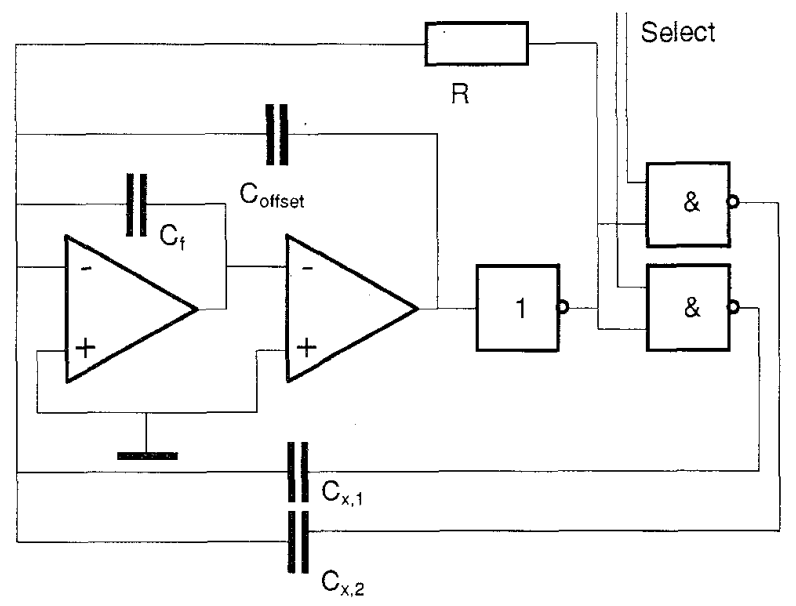

Fig. 3. The modified Martin oscillator.

an imperfect shielding between the input of the charge amplifier and the voltage source. This capacitor does not depend on the selected capacitor and will be eliminated, as we will show later on.

\section{The Capacitance-Controlled Oscillator}

Martin [7] proposed a switched-capacitor relaxation oscillator with a linear relationship between the capacitance and the period. In our system the switched capacitor has been replaced by a resistor [8] (Fig. 3). The period of the oscillator amounts to $[1]$

with

$$
T=4 R\left(C_{o f f s e t}^{\prime}+C_{x, i}\right), \quad(i=1,2)
$$

$$
C_{o f f s e t}^{\prime}=C_{o f f s e t}-C_{o s}
$$

\section{The Three-Signal Approach}

To eliminate the unknown values $C_{\text {offset }}^{\prime}$ and $R$ in (1), a continuous auto-calibration process called the three-signal approach [6] has been applied. The idea is to perform three successive measurements $\left(C_{x 1}, C_{x 2}\right.$, and $\left.C_{o f f s e t}^{\prime}\right)$ in exactly the same way. When a linear relationship is assumed between the measured periods $\left(M_{x 1}, M_{x 2}, M_{o f f s e t}\right)$ and the

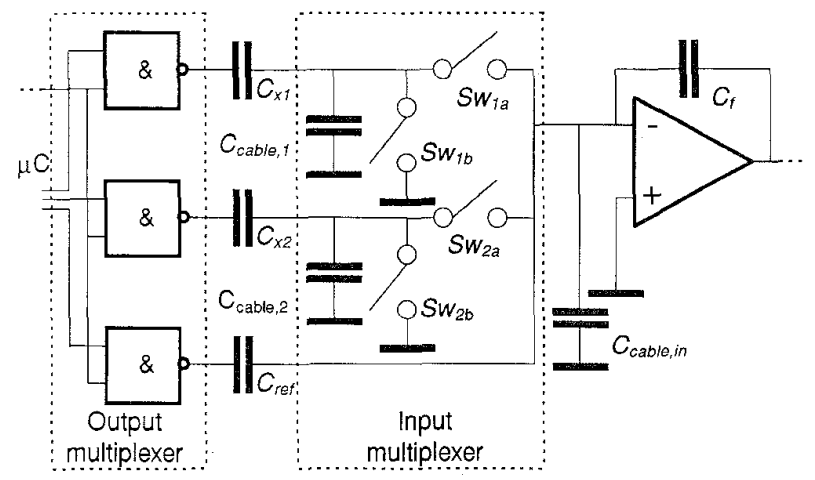

Fig. 4. Adding multiplexers to the input.

TABLE I

Multiplexer Settings During 3-Signal Measurement

\begin{tabular}{ccccc}
\hline Measurand & Output-multiplexer & Input-multiplexer & Period & Result (eq. 3) \\
\hline$C_{\text {offset }}$ & Off & $C_{x, i}$ & $T_{\text {offseti }}$ & $M_{\text {offsei }}$ \\
$C_{r e f}$ & $C_{r e f}$ selected & $C_{x, i}$ & $T_{r e f, i}$ & $M_{x 2}$ \\
$C_{x}$ & $C_{x, i}$ selected & $C_{x, i}$ & $T_{x, i}$ & $M_{x l}$ \\
\hline
\end{tabular}

measurands, the capacitance ratio between $C_{x 1}$ and $C_{x 2}$ can be calculated from

$$
\frac{C_{x 1}}{C_{x 2}}=\frac{M_{x 1}-M_{o f f s e t}}{M_{x 2}-M_{o f f s e t}} .
$$

\section{E. Double-Sided Multiplexing}

Adding multiplexers to the input does not necessarily reduce the circuit performance, since the $\mathrm{ON}$ resistances of the switches are negligible and the parasitic capacitances only add to the (much larger) cable capacitances. However, the three-signal approach requires all three measurements to be performed in exactly the same way. This means that the total parasitic capacitance as seen from the input of the charge amplifier must not change during the measurements.

This problem can be resolved as shown in Fig. 4. An extra capacitor $C_{r e f}$, which is not multiplexed, has been added. The three measurements are then performed as shown in Table I. Note that in all three measurements the input-multiplexer is in the same position.

\section{NONIDEALITIES}

In the three-signal approach all additive and multiplicative errors that do not change during the three measurements will be eliminated and along with them many errors caused by nonidealities in the electronic circuit. However, errors that cause nonlinearity will not be eliminated.

\section{A. Additive and Multiplicative Errors}

Nonidealities in the circuit that lead to additive and multiplicative errors include the following. 
1) Input Bias Current of the OP Amp: The ratio between period $T^{\prime}$ with a bias current and $T$ without a bias current is

$$
\begin{aligned}
\frac{T^{\prime}}{T} & =1+\left(\frac{I_{\text {bias }}^{2}}{I_{o}^{2}-I_{\text {bias }}^{2}}\right) \\
& =1+\xi
\end{aligned}
$$

where $I_{b i a s}$ is the bias current and $I_{o}$ the integration current. Therefore $I_{b i a s}$ merely causes a multiplicative error in the period. Although the duty cycle will deviate from $50 \%$, it will not affect the measurement.

2) The Input Offset of the OP Amp: It can easily be shown that the influence of the total input-offset voltage leads to a multiplicative error, similar to that in the previous paragraph.

3) Delay Times of the CMOS Inverters: These lead to an additive error which increases the oscillator period $T^{\prime}$ to

$$
T^{\prime}=T+4 T_{d}
$$

where $T_{d}$ is the delay time.

\section{B. Nonlinearity}

Nonidealities in the circuit that lead to nonlinearity include the following.

1) Output Resistance of the CMOS Inverters: The inverters in the output multiplexer form an RC-circuit with a time constant of $\tau_{\text {cable }, i}=R_{o n}\left(C_{x, i}+C_{\text {cable }, i}\right)$. The resulting error is

$$
\varepsilon_{c a b l e, i}=e^{-(T / 2) / \tau_{c a b l e, i}} .
$$

For an error smaller than $10 \mathrm{ppm}$, the maximum $C_{c a b l e, i}$ can be calculated from

$$
12 \tau_{\text {cable }, i}<\frac{T}{2}
$$

For instance, with $R_{o n}=100 \Omega$ and $T=100 \mu \mathrm{s}, C_{c a b l e, i}$ must be smaller than $41 \mathrm{nF}$.

2) Input Cable Capacitance and the Bandwidth of the $O P$ Amp: The parasitic capacitance $C_{c a b l e, \text { in }}$ forms a voltage divider with $C_{f}$, which reduces the effective bandwidth of the op amp. This causes an HF time constant

$$
\tau_{\mathrm{HF}} \approx \frac{C_{\text {cable, }, i n}}{2 \pi f_{T} C_{f}}
$$

that must satisfy (7), with $\tau_{\text {cable, } i}$ substituted by $\tau_{\mathrm{HF}}$. For instance, with $f_{T}=2 \mathrm{MHz}$ and $C_{f}=20 \mathrm{pF}, C_{c a b l e, \text { in }}$ must be smaller than $1 \mathrm{nF}$.

3) DC Gain of the OP Amp: The DC gain $(A)$ in combination with $C_{f}$ and $R$ causes an $\mathrm{LF}$ time constant at $\tau_{\mathrm{LF}}=$ $A R C_{f}$. For a $10 \mathrm{ppm}$ error this must satisfy

$$
\tau_{\mathrm{LF}}>10^{5} \mathrm{~T}
$$

4) Slewrate: With bipolar op amps or when a switched resistor is used as current source, the equivalent input resistance in combination with the slew rate of the op amp causes a relative error

$$
\varepsilon_{\text {slew }} \approx \frac{V_{c c}}{4 R C_{f} S_{R}} \cdot \frac{C_{\text {ref }}}{C_{f}+C_{\text {offset }}+C_{\text {par }}}
$$

where $V_{c c}$ is the supply voltage and $S_{R}$ the slew rate. Adding series resistances to $C_{x}$ and $C_{\text {offset }}$ prevents slewing and minimizes this error.

5) Comparator: The comparator delay is often modeled as a constant. However, measurements have shown this delay to be dependent on the oscillator period, which can lead to nonlinearity.

\section{Noise}

Noise can originate either from the oscillator (thermal noise, etc.) or from using a microcontroller to measure the period (quantization noise).

1) Noise Originating From the Oscillator: Van der Goes [9] shows that the jitter equals

$$
\varepsilon_{n i} \approx \sqrt{\frac{C_{c a b l e, \text { in }}}{C_{x}^{2}} \frac{2 f_{T} C_{f} T v_{e q}^{2}}{V_{c c}^{2} T_{\text {meas }}}}
$$

in which $f_{T}$ is the bandwidth of the op amp, $v_{e q}$ the equivalent input noise $\left(\mathrm{V} / \mathrm{Hz}^{1 / 2}\right)$ of the op amp (assumed to be white noise), $T_{\text {meas }}$ the measurement time and $C_{c a b l e, \text { in }}$ the total input capacitance.

2) Quantization Noise Caused by the Microcontroller: During $N_{o s c}$ periods of the oscillator $N_{\mu C}$ periods of the microcontroller are counted. The relative quantization noise can then be calculated from [10]

$$
\varepsilon_{q n t}=\frac{1}{\sqrt{6}} \frac{T_{c l k}}{T_{\text {meas }}}
$$

where $T_{c^{i k}}$ is the period of the clock.

\section{EXPERIMENTAL RESUltS}

The complete system is shown in Fig. 5. Both the integrator and the comparator have been implemented with an LT1013, which has a bandwidth of $500 \mathrm{kHz}$ and a slew rate of $0.5 \mathrm{~V} / \mu \mathrm{s}$. The oscillator is connected to the programmable counter array of the INTEL 87C51FA microcontroller, which has a $3 \mathrm{MHz}$ clock.

The measured integrator output voltage with $C_{r e f}$ selected is shown in Fig. 6. Several measurements have been performed on the system to determine the offset, nonlinearity and resolution.

The offset can be calculated by not connecting any of the capacitors $C_{x, i}$ to the system and then performing the three-signal measurement. The measured offset was $-40 \mathrm{aF}$. This offset appears to originate from the imperfect shielding between points $\mathrm{A}$ and $\mathrm{B}$ in Fig. 5 . 


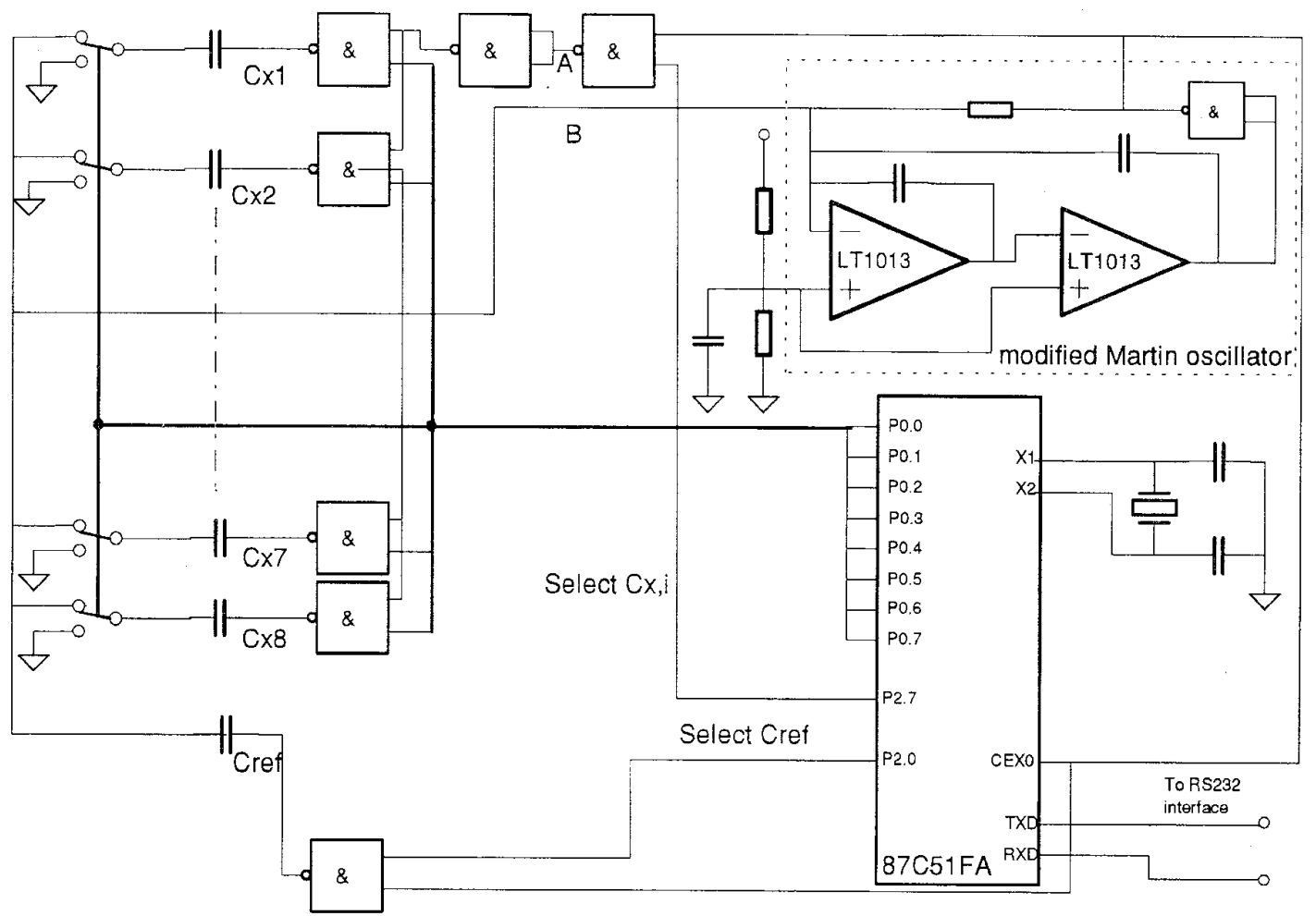

Fig. 5. The complete cireuit.

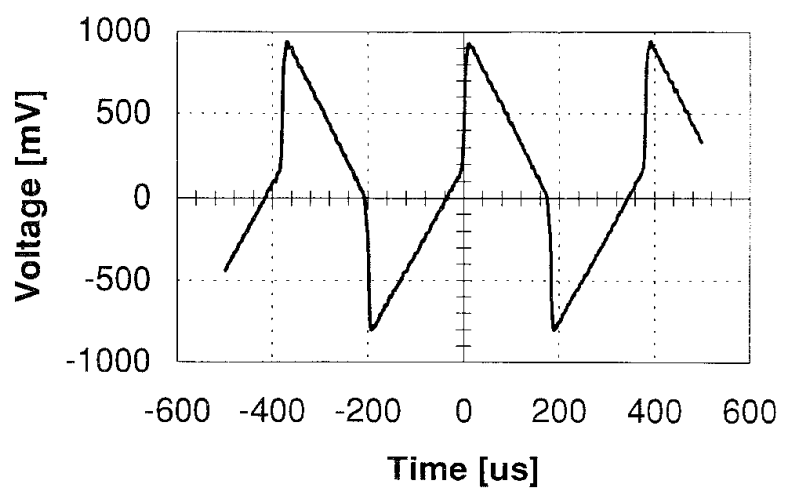

Fig. 6. Integrator output voltage.

The nonlinearity measured over a $2 \mathrm{pF}$ range was found to be less than $100 \mathrm{ppm}$ of the full scale.

The resolution was determined by connecting a capacitance of approximately $1 \mathrm{pF}$ to the system, using two $1 \mathrm{~m}$ coaxial cables of $1 \mathrm{~mm}$ in diameter (AXON), which has a capacitance of $50 \mathrm{pF} / \mathrm{m}$. The capacitance was then measured 100 times by the system. Fig. 7 shows the standard deviation of the capacitance ratio at various measurement times. At $T_{\text {meas }}=$ $100 \mathrm{~ms}$, the standard deviation corresponds to $50 \mathrm{aF}$.

Because of the continuous auto-calibration, the absolute accuracy of the measurement system nearly equals the nonlinearity, provided that a good reference capacitor is available. A low-cost highly stable reference capacitor has been given in [11]. For absolute accuracy this reference capacitor is calibrated against a standard.

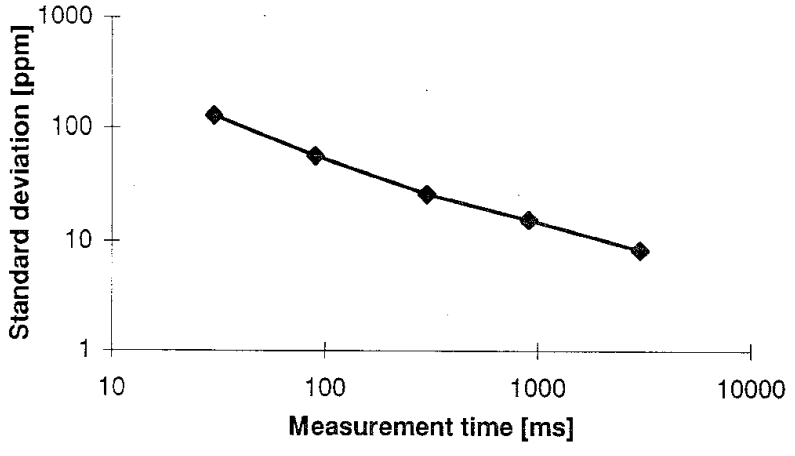

Fig. 7. Standard deviation of the capacitance ratio as a function of the measurement time.

\section{CONCLUSION}

In this paper a very low-cost and accurate capacitancemeasurement system has been presented. Since the system can be integrated on a chip or built from readily available components, the economical production of both small and large quantities is possible. Not only does the measurement system allow for the connection of multiterminal capacitors, but the accuracy has been improved compared to an earlier design. Several have been built and tested, with excellent results for

- offset: $-40 \mathrm{aF}$,

- linearity: $100 \mathrm{ppm}$ over a $2 \mathrm{pF}$ range,

- accuracy: less than $100 \mathrm{ppm}$ over a $2 \mathrm{pF}$ range when an accurate reference capacitor is used, and 
- resolution: 50 aF with a total measurement time of $300 \mathrm{~ms}$ per capacitor, including the offset and reference measurement.

\section{ACKNOWLEDGMENT}

The authors wish to thank Marcus Bonse of the Faculty of Mechanical Engineering for the valuable discussions and his suggestion to use double-sided multiplexing as shown in Section II.

\section{REFERENCES}

[1] F. N. Toth, G. C. M. Meijer, "A low-cost smart capacitive position sensor," IEEE Trans. Instrum. Meas., vol. 41, no. 6, pp. 1041-1044, Dec. 1992.

[2] M. R. Wolffenbuttel, "Surface micromachined capacitive tactile image sensor," Ph.D. Dissertation, Delft University of Technology, 1994.

[3] M. H. W. Bonse, J. W. Spronck, and F. Zhu, "A new two-dimensional capacitive position transducer," Sensors and Actuators A, vol. 41-42, pp. 29-32, 1994.

[4] W. C. Heerens, "Basic principles in designing highly reliable multiterminal capacitor sensors and performance of some laboratory test models," Sensors and Actuators A, vol. 3, pp. 137-148, 1982/1983.

[5] G. W. de Jong, A. M. M. Aalsma, A. J. M. Bertels, K. van der Lingen, G. C. M. Meijer, and J. W. Spronck, "A smart capacitive absolute angularposition sensor," Sensors and Actuators A, vol. 41-42, pp. 212-216, 1994.

[6] G. C. M. Meijer, J. van Drecht, P. C. de Jong, and H. Neuteboom, "New concepts for smart signal processors and their application to PSD displacement transducers," Sensors and Actuators A, vol. 35, pp. 23-30, 1992.
[7] K. Martin, "A voltage-controlled switched-capacitor relaxation oscillator," IEEE J. Solid-State Circuits, vol. SSC-16, no. 4, pp. 412-413, Aug. 1981.

[8] J. van Drecht, "Relaxatie oscillator," Pat. Appl. 91.01076, The Netherlands, 1991.

[9] F. M. L. van der Goes, "Low-cost sensor interfacing," Ph.D. thesis, Delft University of Technology, to be published, 1996.

[10] A. B. Carlson, Communication Systems. New York: McGraw-Hill, 1986 , ch. 12, p. 437.

[11] F. N. Toth, A. J. M. Bertels, and G. C. M. Meijer, "A low-cost highly stable reference capacitor for capacitive sensor systems," in IMTC' 95 , Proc., Waltham, MA, Apr. 24-26, 1995, pp. 412-415.

Ferry N. Toth, for a photograph and biography, see this issue, p. 530.

Gerard C. M. Meijer, for a photograph and biography, see this issue, p. 530.

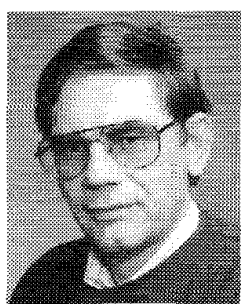

Harry M. M. Kerkvliet was born in Voorburg, The Netherlands, on March 18, 1945. He graduated in electronic engineering at the Royal Polytechnic Institute PBNA, Arnhem, The Netherlands, in 1974. He joined the Department of Electrical Engineering of the Delft University of Technology in 1968, and was involved in areas of television signal processing techniques and electronic system design. His current interests include signal processing. 